\title{
The study of water-head measurement shown in a single tube manometer by use of easy image-processing technique
}

\author{
Yoshifumi Yokoi ${ }^{1, *}$ \\ ${ }^{1}$ National Defense Academy of Japan, Department of Mechanical Engineering, 1-10-20 Hashirimizu, Yokosuka 239-8686, Japan
}

\begin{abstract}
This study proposes the technique which can perform pressure measurement by the low flow velocity which is performed by flow visualization. The technique is the following. The water level image accompanied by the scale shown in a single tube manometer is photographed with a digital camera or a digital camcorder. And image-processing measurement of the image data is carried out in the length of the water level by computer. As verification of the technique, the measurement comparison result of the pressure distribution on the surface of a circular cylinder set in the stream was compared with the previous result. The result was good. It proved that the technique is measurable also by a minute water-head. Furthermore, examination was performed about the validity, the reliability, and the operativity of the technique. As a result, even if who did operation of image-processing measurement, it was shown that high resolution and good accuracy are obtained.
\end{abstract}

\section{Introduction}

In order to understand a "flow", it is important to get to know a flow pattern and pressure. "Flow visualization" is the useful technique of the ability to command a whole view of the flow pattern. In the case of the comparatively low flow velocity, the flow visualization is performed in many cases. On the other hand, although "pressure gauges", such as a manometer, is used in pressure measurement, water-head enough in the low flow velocity for reading is not obtained. Owing to, it is very difficult for the pressure measurement. Therefore, as for "flow visualization" and "pressure measurement", it is common to be carried out by the different flow velocity. It lacks reliability. In order to raise reliability, the appearance of equipment and a technique which can perform pressure measurement by the low flow velocity which is performed by flow visualization is required.

The water-head obtained by the low flow velocity is very little. In order to improve reading accuracy of the height of the water-head, expansion of a reading scale is effective. The method of depending on use of a subscale (Vernier scale), expansion with a lens, and optical projection as a method of expanding a reading scale is used. Use of the subscale (Vernier scale) is a technique used for "slide calipers" etc. The method for use looks at the display of the place whose gradation of a subscale and gradation of the main scale which divide a length of $20 \mathrm{~mm}$ into 19 equally corresponded. The resolution (the minimum measurement length) is $0.05 \mathrm{~mm}$. The expansion with a lens provides an expansion lens in the scale section. The technique is introduced into the "Goettingen type manometer."[1] The expansion by optical projection is the method of applying light to the transparent scale hung by the float inside a manometer apparatus, and projecting and expanding to the surface of a wall. The technique is introduced into the "Betz type manometer."[2] The operation fluid of the "Goettingen type manometer" and the "Betz type manometer" is gas, and they are used for observation of an air flow. When operation fluid is a liquid (water), the single tube manometer and $U$ tube manometer from which the end is released by the atmosphere are used. In these manometers, measurement of a water-head will read the gradation of the scale put side by side. The resolution (the minimum measurement length) of the scale is $0.5 \mathrm{~mm}$ visually, and resolution is $0.05 \mathrm{~mm}$ even if it attaches the subscale. Even if it makes 30 degrees of the manometers itself incline, resolution only changes twice. The expansion technique by optical projection is infinitely expandable theoretically. However, the possibility is scarce. An author's research group suggests the technique which raises the resolution of measurement by expanding an image. The technique is the following. A photograph is taken using a digital camera or a digital camcorder with scale gradation in the water level shown by the single tube manometer, and image data is obtained. Image processing of the image data is carried out by computer, and the length of a water level is measured. Since this technique is digital measurement, there are few errors by reading. Since the application software installed in the computer is used, an operator can use it without special technical knowledge. And operators' personal ability gap does not appear easily.

In this report, the surface pressure distribution of single circular cylinder is measured using the proposal technique, and having verified as compared with the 


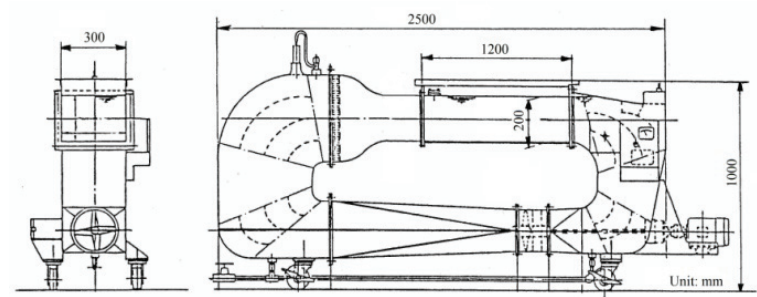

Fig. 1. Schematic diagram of closed circuit water channel

previous experimental result [3], the measurement accuracy, and reliability are described.

\section{Experimental apparatus and method}

\subsection{Outline of experimental apparatus}

The experimental apparatus consists of a small closed circuit water channel, a pressure measuring device, and a video recording apparatus. The closed circuit water channel apparatus is a small water tank with a water capacity of $0.4 \mathrm{~m}^{3}$, and the size of test section of water tank are length of $1.2 \mathrm{~m}$ and cross-section of $0.3 \mathrm{~m} \times 0.3 \mathrm{~m}$ square meters. The test section is an open channel. The board for circular cylinder attachment is prepared in the open section of the test section. The line which shows a direction is drawn on the board. It is the mechanism in which direction of a pressure hole can be set by piling up the line and mark of a circular cylinder end. The sketch of the closed circuit water channel is shown in Fig. 1. At the time of the experiment, the water level was set as $0.2 \mathrm{~m}$. The stream is generated by carrying out input operation of the operation frequency $f \mathrm{~Hz}$ in a digital inverter. The stream velocity $v$ is obtained by $v=0.0327 \times$ $f(\mathrm{~Hz})$.

The pressure measuring device consists of a circular cylinder and a single tube manometer. The diameter of the circular cylinder is $10 \mathrm{~mm}$, and the cylinder length is $300 \mathrm{~mm}$. The screw for setting is prepared in the upper edge of the circular cylinder, and the marker in which a direction is shown is describing in the edge. The small hole which is the diameter of $1 \mathrm{~mm}$ for pressure measurement is provided in the position which is $100 \mathrm{~mm}$ from the circular cylinder lower end. It connects with the single tube manometer lower end by a vinyl tube through the taxiing way provided in the circular cylinder from the small hole. The aspect of the circular cylinder for pressure measurement is shown in Fig. 2. A single tube manometer and the overall apparatus are shown in Fig. 3. The manometer main part is glass and uses a little thick tube (the diameter of inner is $10 \mathrm{~mm}$ ) in consideration of the surface tension of water. The scale made from the steel for water level reading is attached in the side of the glass tube.

The video recording apparatus consists of a camera for photography, and a personal computer for image processing. The camera for photography used the digital camera (Nikon D40) and the digital camcorder (WAT250D2) properly by the use. Those cameras fixed to the

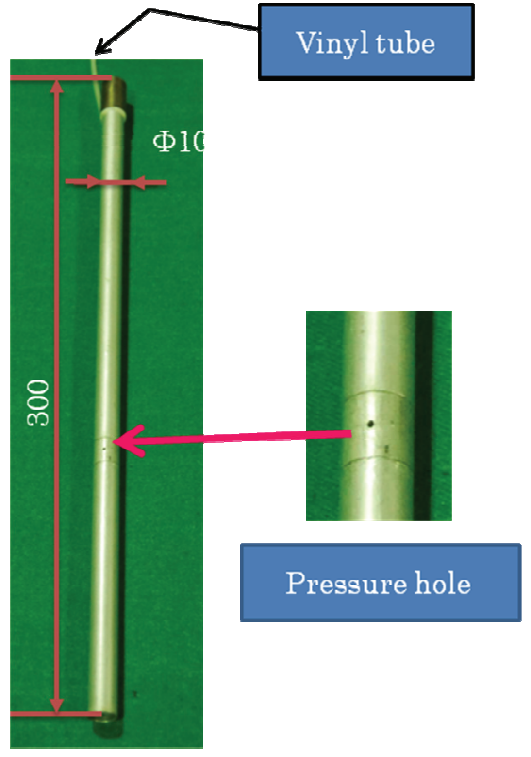

Fig. 2. Test circular cylinder for pressure measurement

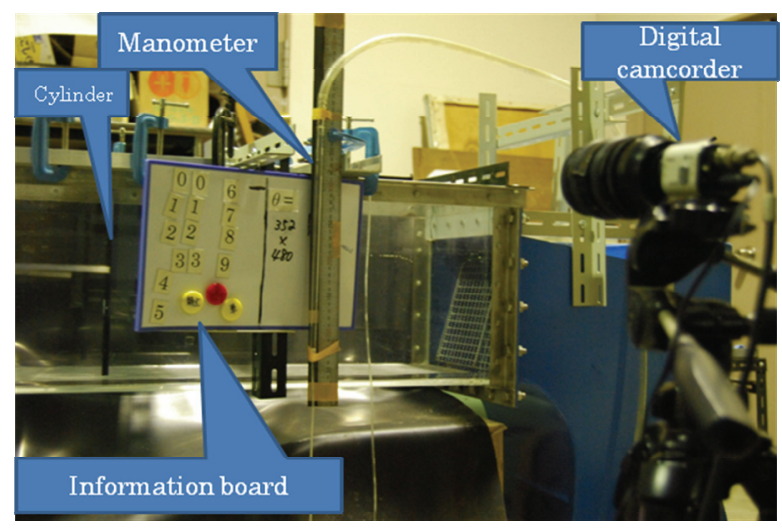

Fig. 3. The test section and single tube manometer of the closed circuit water channel apparatus, and digital camcorder arrangement

tripod mount were set in the manometer front. The personal computer (NEC LaVie) commercial note type was used for image-processing measurement. Measurement of the length of picture was performed using "Microsoft Paint" [3] installed as application software. The video signal converter (Alterplus AX-200) was used for dealing with the image signal from a digital camcorder with a personal computer.

\subsection{Experimental procedure}

The experimental procedure is the following. Water is filled in a closed circuit water channel, and the water level of the test section is adjusted to $200 \mathrm{~mm}$. Warming up is performed. A test circular cylinder is set in a test section. The pressure tube from the circular cylinder is connected to the single tube manometer. The focus and zoom of the photography camera are adjusted. Those conditions are held after completing this adjustment until a series of photography is completed. The water level of 
the manometer in a stream state of rest is photographed. The stream is generated. The water-head of the manometer in each direction angle of the circular cylinder is photographed and recorded. The obtained picture is downloaded to the computer, the imageprocessing measurement is performed, and a pressure coefficient $C_{P}$ is calculated.

\subsection{Procedure of image acquisition for measurement}

It is set as 0 degree of directions, and the manometer water level at the time of stream stationary is photographed. Warming up operation is performed for 3 minutes, and the manometer water-head at the time of 0 degree of directions is photographed. The next circular cylinder direction is set up after the photography, and the manometer water-head after warming up operation for 3 minutes is photographed. The operation is performed to 360 degrees (going around 0 degrees) of directions. It confirms that the manometer water-head of 0 degree of directions and 360 degrees of directions becomes the same. And the value also confirms that it is the maximum. The photographed image data is introduced into the computer. When the digital camera was used, the work was performed through memory card (SD card). When the digital camcorder was used, the work was performed through the USB conversion unit.

\subsection{Procedure of the water-head length measurement by image processing}

The "Paint" is started and the target picture file is opened. A reference position is decided in the displayed picture. Here, a reference position uses the same position over a series of measurement. A square is chosen from the "figure" in the section of a menu display of the software screen upper part. The displayed square is drawn on the manometer and scale. It meets with the reference position which determined one of the sides of the square. Another side is met with the manometer water-head position. For example, the upper side will be met with the manometer water-head position if the base of the square is met with the reference position. Since the information on the length of two sides of the square (the number of horizontal pixels, $\mathrm{x}$, the number of vertical pixels) is displayed on the software screen bottom, the information on the number of vertical pixels is read. The actual dimension per pixel is obtained by authorization. For example, it is investigated by the box function whether an actual length of $10 \mathrm{~mm}$ shown on the screen is displayed with how much number of pixels. The thickness of the drawing line sets up so that it may be easy to operate it.

\subsection{Distribution of pressure coefficient $C_{P}$ on surface of circular cylinder}

The procedure which measures the pressure distribution on the surface of a circular cylinder is written in the following. A reference position is determined as the scale shown on the screen. It is set as 0 degree of directions, and the length between the water level and the reference position at the time of stationary is measured. The length is called $l_{0}$. A stream is generated and the length between the manometer water-head for every setting angle and the reference position is measured. Those length is called $l_{\theta}$. The pressure coefficient $C_{P}$ is calculated by $\left(l_{\theta}-l_{0}\right) /\left(\rho v^{2} / 2\right)$.

\subsection{Experimental parameters}

The experimental parameters for obtaining pressure distribution are the flow velocity and an angle of direction. The flow velocity was made into $v=0.25 \mathrm{~m} / \mathrm{s}$ and $0.98 \mathrm{~m} / \mathrm{s}$ so that the data of previous papers could be referred to. And also when the flow velocity is $0.25 \mathrm{~m} / \mathrm{s}$, according to Bernouilli's theorem, an anticipation waterhead is $3.2 \mathrm{~mm}$. An angle of direction is every 10 degrees in the range of 0 to 360 degrees. The parameters for investigating the reliability of the measurement technique are "resolution", "magnification", "human deviation", and "the thickness of line." The resolution is seven kinds. The magnification is four kinds. The human deviation investigates whether a human element influences image-processing measurement. The investigation had the same operation given to five persons, and the difference was investigated. The thickness of the line at the time of writing the "box" is two kinds. They are the case of the minimum width, and the case of the width accompanied by a central line. The experiment parameters are shown in Table 1.

Table 1. Experimental parameter

\begin{tabular}{|c|c|c|}
\hline Item & Number & Contents \\
\hline Reynolds number & 2 & $R e=2500,9800$ \\
\hline Angle of direction & 36 & 0 degree to 360 degrees \\
\hline Resolution & 7 & $\begin{array}{c}174 \times 144,320 \times 240,352 \times 240,640 \times 480, \\
720 \times 480,480 \times 480,352 \times 480\end{array}$ \\
\hline Magnification & 4 & $100 \%, 200 \%, 300 \%, 400 \%$ \\
\hline Human deviation & 5 & Student \\
\hline
\end{tabular}




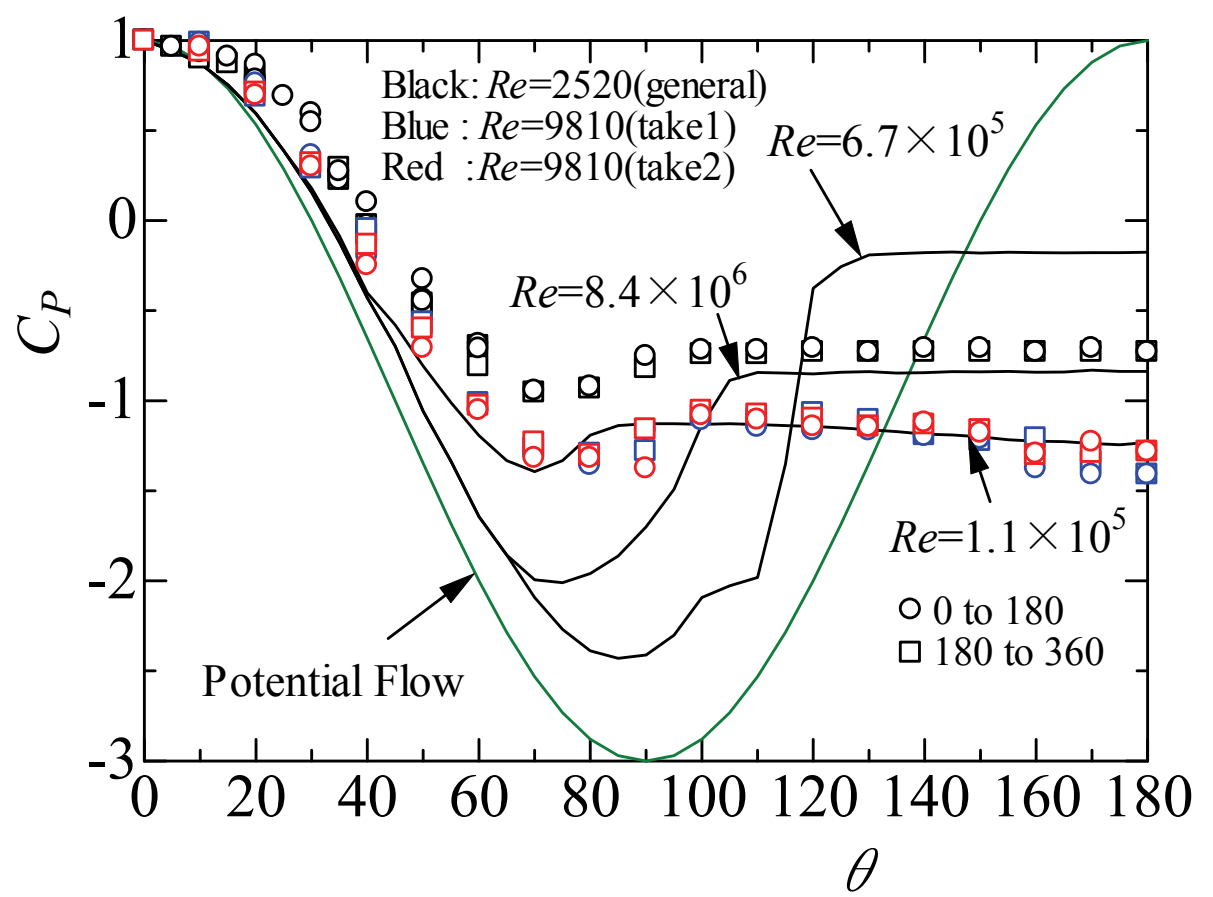

Fig. 4. The pressure coefficient distribution on the surface of a circular cylinder; comparison with the results measured by this technique, and previous measurement results [4]

\section{Experimental results and discussion}

\subsection{Pressure distribution on surface of circular cylinder}

The measurement result of the pressure on the surface of a circular cylinder using the suggested technique is shown in Fig. 4. An abscissa is the angle of direction and an ordinate is the pressure coefficient. As for the experimental data shown in Fig. 4 here, data is added partially every 5 degrees. The result (black) in Reynolds number 2500 and the result (red and blue) in Reynolds number 9800 are shown. As for the previous result (in the case of a potential flow or high Reynolds number) [4] is shown for comparison. The circle in a figure $(\mathrm{O}$ symbol) is the case of 0 to 180 degrees, and the square mark ( $\square$ symbol) is the case of 180 to 360 degrees. It is seen that the symmetry of pressure distribution is good. When Reynolds number is $R e=2500$, as for the pressure distribution on the surface of circular cylinder, as compared with other cases, the pressure loss seems to be little. Here, the minimum $C_{P}$ value is obtained at about 70 degrees. And the position of zero crossing is about 40 degrees. In the potential flow, the position of zero crossing is 30 degrees. A little scatter is seen in 30 to 60 degrees. It seems that this is based on the size of the pressure hole. It is because this section has a large change of pressure by an angle. The diameter of the circular cylinder is $10 \mathrm{~mm}$, and the diameter of the pressure hole is $1 \mathrm{~mm}$. So, it seems that the size of a pressure hole has influenced pressure measurement. Accordingly, it seems that it is not an error of measurement by the suggested measurement technique. When Reynolds number was $R e=9800$, it was obtained that it is well in agreement as compared with previous experimental data $R e=1.1 \times 10^{5}$. It supports the usefulness of this measurement technique that it was in agreement with the known profile by comparable Reynolds number. The repeatability of the experiment is also considered to be a good thing. In the case of the low flow velocity, enough water-head to measure is not obtained. In the case of Reynolds number 2500, the water-head obtained by the maximum pressure (total pressure, position of 0 degree) is about $3 \mathrm{~mm}$. By such the state, measurement data was not obtained in accuracy enough in the previous measurement technique. However, it proves that this technique is suitable for small pressure measurement that the exact profile was obtained by use of this technique.

\subsection{Accuracy and reliability over the image- processing measurement}

The selling point of proposal technique is the following. Anyone can carry out easily, and the same result is obtained even if who carries out. There is no use of special software and it can carry out cheaply. In order to demonstrate this thing, the experiment which changes an operator and measures the length of the scale on a picture using the same picture was performed. The measurement result is shown in Table 2. At this time, the number of pixels was $640 \times 480$ (horizontal $\times$ vertical), and display magnification was $100 \%$. If the 
Table 2. Comparison of the measurement result by the operator in length measurement

\begin{tabular}{|c|c|c|}
\hline Length & Operator A & Operator B \\
\hline $10 \mathrm{~mm}$ & 46 & 46 \\
\hline $20 \mathrm{~mm}$ & 90 & 90 \\
\hline $30 \mathrm{~mm}$ & 134 & 135 \\
\hline $40 \mathrm{~mm}$ & 179 & 180 \\
\hline $50 \mathrm{~mm}$ & 222 & 222 \\
\hline
\end{tabular}

Table 3. Comparison of the measurement result by the operator in the length measurement when changing the thickness of the line

\begin{tabular}{|c|c|c|c|}
\hline Length & Operator C & Operator D & Operator E \\
\hline $10 \mathrm{~mm}$ & 45 & 45 & 46 \\
\hline $20 \mathrm{~mm}$ & 90 & 90 & 90 \\
\hline $30 \mathrm{~mm}$ & 134 & 134 & 134 \\
\hline $40 \mathrm{~mm}$ & 178 & 180 & 178 \\
\hline $50 \mathrm{~mm}$ & 222 & 222 & 222 \\
\hline
\end{tabular}

Table 4. Comparison of the measurement result by the operator in measurement of the same length when changing magnification

\begin{tabular}{|c|c|c|c|c|c|}
\hline Magnification & Operator A & Operator B & Operator C & Operator D & Operator E \\
\hline $100 \%$ & 134 & 135 & 134 & 134 & 134 \\
\hline $200 \%$ & 134 & 135 & 134 & 134 & 134 \\
\hline $300 \%$ & 134 & 135 & 134 & 134 & 134 \\
\hline $400 \%$ & 136 & 136 & 134 & 134 & 134 \\
\hline
\end{tabular}

measurement result is compared, it can be found that measurement of length shows the same pixel value by each measurement person. When the length of a scale is $30 \mathrm{~mm}$ and $40 \mathrm{~mm}$, only 1-pixel difference can be seen. It is thought that it was produced when the cause of this difference drew a "box" and it dropped on the picture. It seems that the thickness of the line describing a "box" involves. The thinnest line was chosen at this time. Therefore, since it was hard to attach the scale graduation line and distinction to meet, the difference was produced. In $30 \mathrm{~mm}$ measurement, when a 1-pixel difference is based on 134 pixels, it is $0.224 \mathrm{~mm}$ per pixel. In $40 \mathrm{~mm}$ measurement, when a 1-pixel difference is based on 179 pixels, it is $0.223 \mathrm{~mm}$ per pixel. When based on Operator's A result, each resolution is $0.217 \mathrm{~mm}$ per pixel, $0.222 \mathrm{~mm}, 0.224 \mathrm{~mm}, 0,223 \mathrm{~mm}$, and $0,225 \mathrm{~mm}$ to a measuring object length of $10 \mathrm{~mm}, 20 \mathrm{~mm}, 30 \mathrm{~mm}$, $40 \mathrm{~mm}$, and $50 \mathrm{~mm}$.

\subsection{Influence of line thickness}

In order to investigate the influence the thickness of the line of the "box" affects a measurement result, the length of the scale gradation was measured by the thickest line. As a function of software, if the line is made thick, a central line will be displayed. It decided to meet this central line with an object. There is a merit which is easy to distinguish since this line will not assimilate to a picture in back if a line is made thick. The verification result at the time of performing this idea is shown in Table 3. Here, subjects are three persons different from the test of the preceding clause. The experiment is performed on the same conditions as the test of the preceding clause. There is no difference special among three subjects, and having obtained good agreement is found. This result shows the thickness of the line only contributing to the ease of carrying out of operation, and not influencing the result of measurement. What is necessary is just to adjust "thickness of the line" with sufficient convenience to an operator's work.

\subsection{Influence of picture magnification}

In measuring the length on the picture, if picture size is large, it will be expected that operation of measurement becomes easy. When magnification was changed, the influence affect measurement was investigated. The experiment changed magnification for the same picture, and measured the length of $30 \mathrm{~mm}$ of the scale gradation by five subjects. The experimental result is shown in Table 4. It is shown that each measurement person's result does not have the difference by magnification. It is found that the value of the pixel obtained even if it changes the magnification on an image display does not change. Accordingly, what is necessary is just to set up magnification by the ease of carrying out of work.

\subsection{Improvement in resolution}

It seems that it is effective to set up many numbers of pixels at the time of photography in order to raise the resolution. The number of setting pixels was changed at the time of photography, and the same photographic object was photographed. Those photographs are shown in Fig. 5. Here, description of the number of pixels is "horizontal $\times$ vertical." The picture will become large if the number of pixels increases. Reading of the waterhead of the manometer is the number of pixels of the vertical component. Therefore, 144 pixels, 240 pixels, and 480 pixels of the number of pixels of the vertical component are the targets for comparison. Each operator measured the length of $30 \mathrm{~mm}$ of scale gradation about three kinds of the number of vertical component pixels. The result is shown in Table 5. The resolution to the number of pixels of 114,240 , and 480 is $0.750 \mathrm{~mm}$, $0.411 \mathrm{~mm}$, and $0.222 \mathrm{~mm}$, and it is found that resolution improves with the increase in the number of pixels. It is not based on the resolution but there is scatter in measurement between operators. However, an actual 


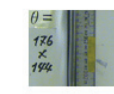

(a) $176 \times 144$

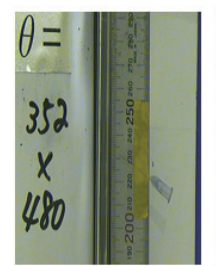

(d) $352 \times 480$

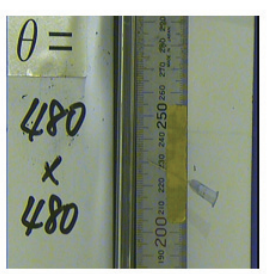

(e) $480 \times 480$
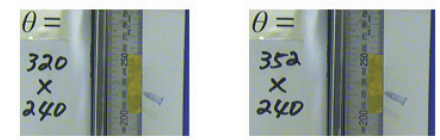

(b) $320 \times 240$

(c) $352 \times 240$

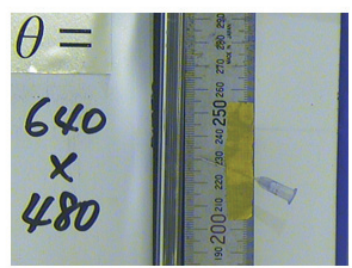

(f) $640 \times 480$

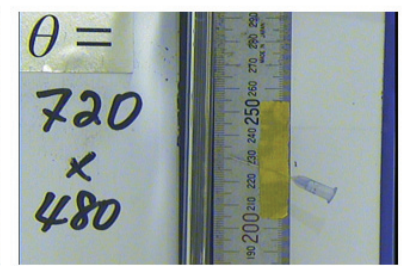

(g) $720 \times 480$

Fig. 5. Magnitude comparison of the image by picture resolution; the number of pixels is the following, respectively, (a)25344pixels, (b)76800pixels,(c)84480pixels, (d)168960pixels, (e)230400pixels, (f)307200pixels, (g)345600pixels

Table 5. Comparison of the measurement result by the operator in measurement of the same length when changing picture resolution

\begin{tabular}{|c|c|c|c|c|c|}
\hline Pixel & Operator A & Operator B & Operator C & Operator D & Operator E \\
\hline 144 & 40 & 40 & 41 & 40 & 40 \\
\hline 240 & 68 & 68 & 67 & 68 & 68 \\
\hline 480 & 135 & 135 & 134 & 134 & 135 \\
\hline
\end{tabular}

error will decrease by raising resolution, since the variation is about 1 pixel.

\section{Conclusions}

In this study, the pressure measurement in the low flow velocity suggested the technique which can be easily performed with sufficient accuracy. Surface pressure of the circular cylinder was measured as the example of verification. The following conclusions were obtained.

(1) Even when enough water-head for reading was not obtained, it became clear that it was the technique which can measure in enough accuracy.

(2) It was found that this technique has few human errors of measurement.
(3) In order to raise resolution, it is important to increase the number of pixels at the time of photography.

(4) The resolution will become high if the number of pixels of the direction of the target measurement is increased.

\section{References}

1. K. Suzuki, S. Fujita, H. Tsujikawa, The Chemical Society of Japan, 30-4, 321-322 (1982)

2. K. Komiya, Turbomachinery, 4-9, 551-557 (1976)

3. https://en.wikipedia.org/wiki/Microsoft_Paint

4. T. Toyokura, K. Kamemoto, Fluid Dynamics, (1981), 234, Jikkyo Pres.

5. Y.Yokoi, R. Vitkovičová, EPJ Web of Conf. 143, 02146 (2017) 